\title{
Modulation of store-operated calcium entry and nascent adhesion by p21- activated kinase 1
}

\author{
In-Sook Jeon ${ }^{1}$, Hye-Ryun Kim', Eun-Young Shin' , Eung-Gook Kim', Heon-Seok Han², Jin-Tae Hong ${ }^{3}$, Hak-Kyo Lee , \\ Ki-Duk Song (i) $^{4}$ and Joong-Kook Choi ${ }^{1}$
}

\begin{abstract}
Calcium mobilization is necessary for cell movement during embryonic development, lymphocyte synapse formation, wound healing, and cancer cell metastasis. Depletion of calcium in the lumen of the endoplasmic reticulum using inositol triphosphate (IP3) or thapsigargin (TG) is known to induce oligomerization and cytoskeleton-mediated translocation of stromal interaction molecule 1 (STIM1) to the plasma membrane, where it interacts with the calcium release-activated calcium channel Orai1 to mediate calcium influx; this process is referred to as store-operated calcium entry (SOCE). Furthermore, aberrant STIM1 or SOCE regulation is associated with cancer cell motility and metastasis. The p21-activated kinases (PAKs), which are downstream effectors of GTPases, reportedly regulate cytoskeletal organization, protrusive activity, and cell migration. Although cytoskeletal remodeling apparently contributes to calcium mobilization via SOCE, and vice versa, the mechanisms by which they regulate each other remain unclear. In this study, we aimed to characterize whether PAK1 modulates calcium mobilization and STIM1 localization. Our data demonstrate that PAK1 interacts with STIM1 in vitro and that this interaction was enhanced by treatment with a nascent adhesion inducer, such as phorbol 12,13-dibutyrate (PDBu). Under basal conditions, both proteins appeared to primarily colocalize in the cytosol, whereas treatment with PDBu induced their colocalization to vinculin-positive peripheral adhesions. Downregulation of PAK1 activity via chemical inhibitors or by PAK1 shDNA expression impaired STIM1-mediated calcium mobilization via SOCE. Based on these findings, we propose that PAK1 interacts with STIM1 to regulate calcium mobilization and the formation of cellular adhesions.
\end{abstract}

\section{Introduction}

Intracellular calcium $\left(\mathrm{Ca}^{2+}\right)$ levels are precisely controlled in non-neuronal cells by ligand-gated $\mathrm{Ca}^{2+}$ ion channels located on the plasma membrane (PM), endoplasmic reticulum (ER), and mitochondria. The activation of PM receptors by growth factors, neurotransmitters, or hormones results in the production of inositol

Correspondence: Ki-Duk Song (kiduk.song@gmail.com) or Joong-Kook Choi (jkchoi@chungbuk.ac.kr)

${ }^{1}$ Division of Biochemistry, Chungbuk National University, City of Cheongju 361763, Korea

${ }^{2}$ Department of Pediatrics, College of Medicine, Chungbuk National University, City of Cheongju 361-763, Korea

Full list of author information is available at the end of the article. triphosphate (IP3) and the subsequent activation of IP3 receptors in the ER, which triggers the release of $\mathrm{Ca}^{2+}$ ions from ER storage $\mathrm{e}^{1-5}$.

Upon depletion of ER $\mathrm{Ca}^{2+}$ stores, the ER membrane protein stromal interaction molecule 1 (STIM1) undergoes a conformational change, resulting in selfoligomerization and translocation to the PM for interaction with the calcium release-activated calcium channel protein Orai $1^{6-8}$. During the translocation process, STIM1 is known to bind to the microtubule (MT) tip attachment protein end-binding protein 1 (EB1), thus regulating the formation and extension of new ER tubules toward the $\mathrm{PM}^{4,9-11}$. ER-PM junctions serve as platforms for lipid exchange between organelles and for cell 
signaling, notably $\mathrm{Ca}^{2+}$ and cAMP signaling ${ }^{12}$. The stability of ER-PM junctions and the store dependence of junction abundance varies among different cell types, as the polymerization of cortical actin, which is thought to interfere with junction formation, inhibits store-operated calcium entry (SOCE) in some cells but not others ${ }^{4,13,14}$. In human disease models, STIM1 and SOCE have been shown to promote the migration or invasion of a diverse array of human cells, including prostate cancer cells ${ }^{15}$, colorectal cancer cells ${ }^{16}$, glioma cells ${ }^{17}$, clear cell renal cell carcinoma cells ${ }^{18}$, endometrial adenocarcinoma cells ${ }^{19}$, and cardiac c-kit ${ }^{+}$progenitor cells ${ }^{20}$.

Rho family GTPases, which include Rac, Cdc42, and Rho, are small GDP/ GTP-binding proteins that regulate several aspects of intracellular actin dynamics and cellular signaling ${ }^{21-23}$. In addition to GDP/GTP exchange, most Rho/Rac proteins must dock onto cell membranes to perform their biological functions ${ }^{24}$. A variety of effector proteins, including kinases, are known to be recruited during this process. Rho-associated serine/threonine kinases include p21-activated kinase (PAK), mixedlineage kinase (MLK), Rho-associated kinase (ROK), myotonic-related Cdc42-binding kinase (MRCK), citron kinase (CRIK), and protein kinase novel (PKN). Among these kinases, the regulation of PAK by Rac1 and Cdc42 is relatively well-characterized ${ }^{25-27}$.

PAK1 has been implicated in signaling pathways associated with cytoskeletal changes, cell motility ${ }^{28,29}$, and focal adhesion ${ }^{30-34}$. Additionally, PAK activity reportedly plays a role in $\mathrm{Ca}^{2+}$ mobilization. Bone marrow mononuclear cells lacking PAK1 exhibit reduced $\mathrm{Ca}^{2+}$ mobilization and altered depolymerization of cortical filamentous actin (F-actin) in response to stimulation of the FceRI IgE receptor ${ }^{35}$. Conversely, PAK2 does not appear to regulate $\mathrm{Ca}^{2+}$ influx and is not enzymatically controlled by $\mathrm{Ca}^{2+}$ influx ${ }^{36,37}$. In a murine cardiac model, PAK1 appears to be an important regulator of sarco/ endoplasmic reticulum $\mathrm{Ca}^{2+}$ ATPase (SERCA) expression in cardiomyocytes for ventricular $\mathrm{Ca}^{2+}$ homeostasis ${ }^{38}$.

Because members of the PAK family of proteins are key players in regulating the intracellular cytoskeleton, and because STIM1/SOCE are involved in cell migration, we sought to investigate whether PAK1 modulated STIM1mediated $\mathrm{Ca}^{2+}$ mobilization and STIM1 localization. Biochemical and confocal imaging assays were used to study the interaction between PAK1 and STIM1, as well as their colocalization. $\mathrm{A} \mathrm{Ca}^{2+}$ mobilization assay involving thapsigargin (TG) was used to determine whether PAK1 activity correlated with SOCE activity level. Finally, we investigated changes in cellular adhesion following phorbol 12,13-dibutyrate (PDBu) treatment in cells, using vinculin as a marker to identify the nature and extent of PAK1 and STIM1 localization.

\section{Materials and methods}

\section{Plasmids and reagents}

Full-length human STIM1 cDNA was PCR-amplified using gene-specific primers and cloned into the cytomegalovirus (CMV) promoter-derived mammalian expression vector pCS4-3xFlag, followed by DNA sequence verification. The cytoplasmic region (amino acids 235-685) and the C1 (amino acids 235-350), C2 (amino acids 341-490), and C3 (amino acids 491-685) STIM1 cDNA regions were cloned into the pGEX-4T-1 plasmid to express and purify cytoplasmic STIM1 GST fusion proteins of varying lengths. Full-length human Orail cDNA was cloned into the pmCherry-N1 vector, followed by DNA sequence verification. Wild type (WT), dominant active (DA), and dominant-negative (DN) PAK1 cDNAs were cloned into $\mathrm{pCMV}$-myc-tagged plasmids as described in Kim et al. ${ }^{39}$. The DA and DN forms of PAK1 in RFP (pDsRed2-N1) plasmids were provided by Eun-Young Shin at Chungbuk National University, Korea.

PAK1 inhibitors [1, 4-naphthohydroquinone (1, 4-NHQ) and PF3758309] were purchased from Sigma-Aldrich (St Louis, MO, USA) and Calbiochem (Sandiego, CA, USA), respectively. PDBu was purchased from Tocris Bioscience (Bristol, UK) and used at a $100 \mathrm{nM}$ concentration for western blot analysis and confocal imaging.

Antibodies against the Myc (9E10; Santa Cruz Biotechnology) and Flag (M2; Sigma) epitopes and vinculin (Abcam, Cambridge, UK) were used for western blot analysis and confocal imaging. Secondary antibodies against Myc and Flag epitopes (goat anti-mouse Alexa Fluor $^{\circledR} 488$ and goat anti-rabbit Alexa Fluor ${ }^{\circledR} 594$ and 633; Invitrogen) were also used. Rabbit monoclonal PAK1 (Abcam, ab40852) and mouse monoclonal STIM1 (Santa Cruz, sc-68897) antibodies were used to detect endogenous PAK1 and STIM1, respectively.

\section{Cell culture and cell lines}

HEK293 and HeLa cells were maintained in Dulbecco's modified Eagle's medium (DMEM) containing 10\% fetal bovine serum (FBS) supplemented with penicillin and streptomycin. For starvation, the cells were transfected with plasmids, the growth media was discarded after $12 \mathrm{~h}$, and the cells were washed once with $1 \times$ phosphate-buffered saline (PBS) and replenished with DMEM containing penicillin and streptomycin but no FBS for the indicated time periods. A PAK1 knockdown (K/D) cell line was established by transfecting HeLa cells with a PAK1 shRNA vector (Origene, pRFP-C-RS), followed by selection for puromycin resistance and western blotting analysis of reduced PAK1 expression using a polyclonal anti-PAK1 antibody (Cell Signaling Technology, 2602S). 


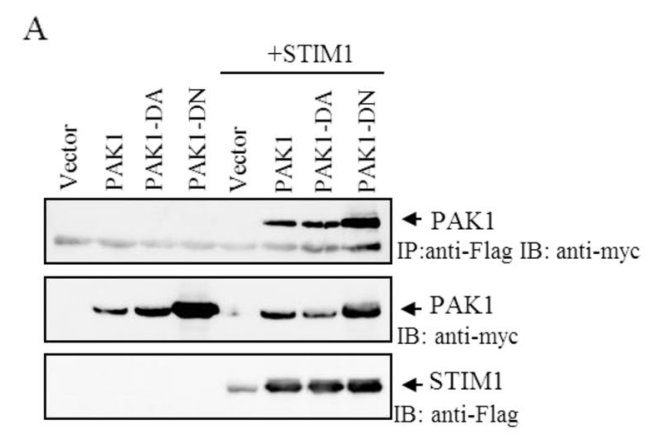

B IP: IgG STIM1 STIM1

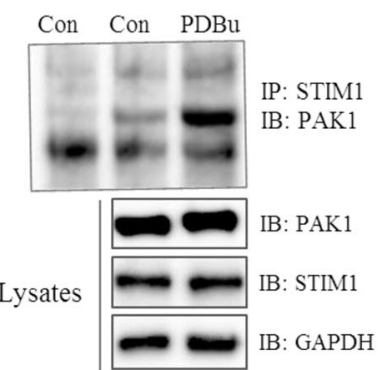

C

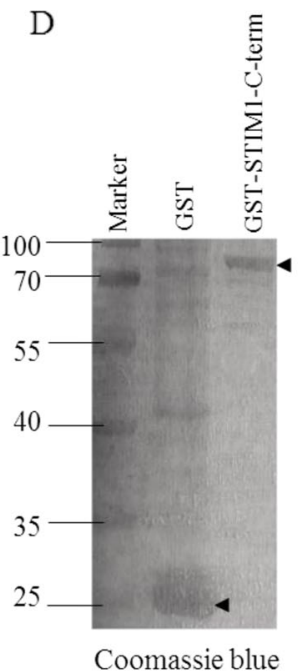

E

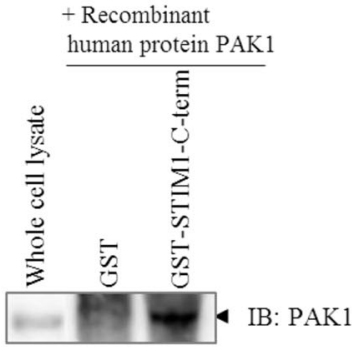

Coomassie blue

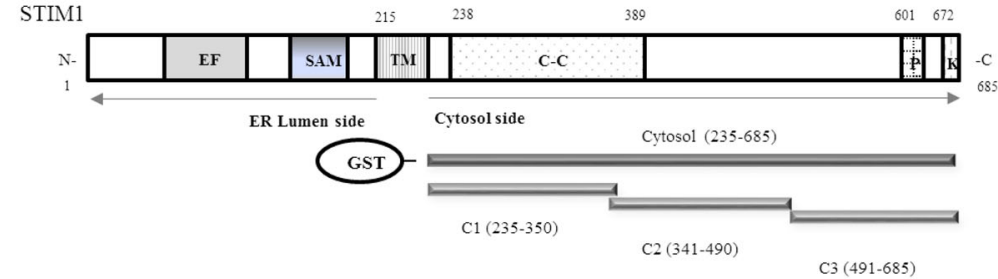

$$
\text { F }
$$

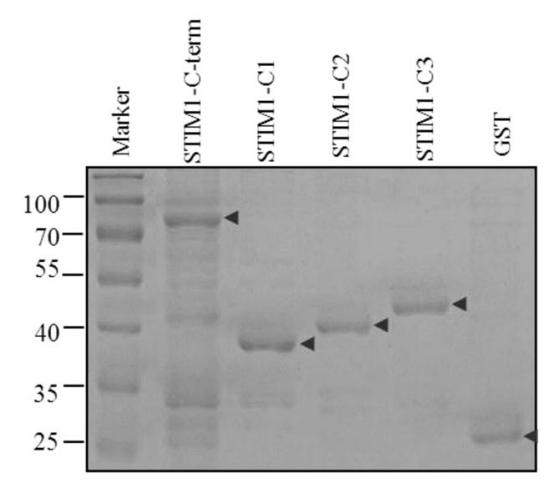

G

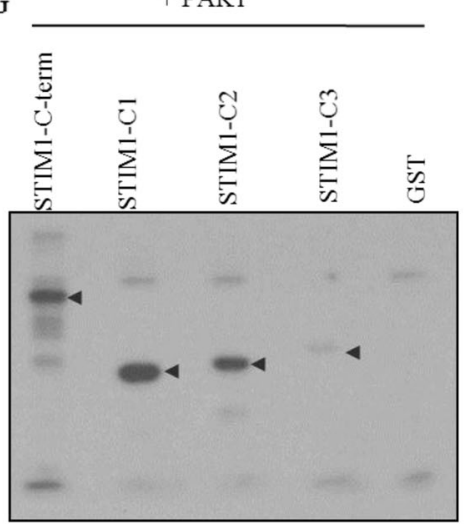

Fig. 1 (See legend on next page.) 
(see figure on previous page)

Fig. 1 PAK1 interacts with and phosphorylates STIM1. HEK293 cells were transfected with the PAK1 plasmid (c-myc epitope tagged) and/or with STIM1 plasmid (flag epitope tagged). After determining the expression of the STIM1 and/or PAK1 proteins (a, middle and bottom rows), the cleared cell lysates were immunoprecipitated with an anti-flag (STIM1) antibody, followed by Western blotting with an anti-c-myc (PAK1) antibody. (a, top row). HeLa cells were treated with $100 \mathrm{nM}$ PDBu for $30 \mathrm{~min}$, and the cell lysates were cleared for immunoprecipitation with an anti-STIM1 antibody and then analyzed by western blotting with an anti-PAK1 antibody (b). The GST-STIM1 and GST proteins were expressed in E. coli and, after purification, were incubated with purified commercial PAK1 protein followed by western blotting with an anti-PAK1 antibody (c, d). The C-terminal cytosolic region of STIM1 or three sub regions of STIM1 were expressed as GST- STIM1 fusion proteins in E. coli (e, f). Radioactive in vitro kinase assays were performed by incubating $1 \mu \mathrm{g}$ of the PAK 1 protein and $2 \mu \mathrm{g}$ of purified GST-STIM1 fusion proteins in kinase buffer containing [ $\mathrm{Y}^{32} \mathrm{P}$ ] ATP for 30 min at $30{ }^{\circ} \mathrm{C}$ before subjection to SDS-PAGE and autoradiography. GST was included as a negative control (g). EF EF hand motif ( $\mathrm{Ca}^{2+}$ binding), SAM STIM1 oligomerization, TM trans-membrane region, C-C coiled coil domain (Orai1 interaction), P proline rich region, K polybasic cluster, GST glutathione S-transferase

Transfection, western blotting, and immunoprecipitation

HEK293 and HeLa cells were seeded in 6-well plates one day prior to transfection using polyethylenimine (PEI; Sigma-Aldrich) and treated as previously described ${ }^{8}$.

\section{In vitro kinase assay}

GST-STIM1 fusion proteins (GST-STIM1-C-term, -C1, $-\mathrm{C} 2$, and -C3) or GST control proteins were purified from E. coli, and an equal amount $(2 \mu \mathrm{g})$ of each GST-chimeric protein was mixed with $1 \mu \mathrm{l}$ of kinase-active PAK1 (PV3820, Thermo Fisher Scientific, MA, USA) in the presence of $\left[\gamma_{-}^{-32} \mathrm{P}\right]$ ATP and kinase buffer (20 mM HEPES pH 7.4, 1 mM DTT, 0.1 mM Na $V_{4} V_{4}, 2$ mM EGTA, 20 $\mathrm{mM} \mathrm{MgCl}_{2}$, and protease inhibitor) for $30 \mathrm{~min}$ at $30^{\circ} \mathrm{C}$. The resulting reaction samples were resolved by SDSPAGE and subjected to autoradiography.

\section{Immunofluorescence microscopy}

The cells were plated in a 4-well dish (NUNC) at a density of $5 \times 10^{3}$ cells/well and incubated overnight. The cells were then transfected as described above, washed with PBS, fixed with $4 \%$ paraformaldehyde for $10 \mathrm{~min}$ at room temperature (RT), permeabilized in $0.1 \%$ Triton X100 in $1 \times$ PBS for $10 \mathrm{~min}$, washed three times with PBS, and blocked with 10\% FBS. To detect STIM1 and PAK1, the transfected cells were incubated with anti-flag and anti-myc antibodies, respectively, for $1 \mathrm{~h}$ at RT, washed with PBS, and incubated for $30 \mathrm{~min}$ with fluorochromeconjugated secondary antibodies (Alexa Fluor ${ }^{\circledR} 488,594$, and 633 conjugated antibodies). After mounting, the cells were analyzed using a laser-scanning confocal microscope (Zeiss LSM710). The localization of endogenous STIM1 and PAK1 was analyzed as described above using monoclonal PAK1 and polyclonal STIM1 antibodies.

\section{Assay for calcium mobilization}

HeLa cells were transfected with DA or DN PAK1 cDNA (PAK1 in pDsRed2-N1 plasmid) and incubated for $12 \mathrm{~h}$. Then, the cells were incubated with $1 \mu \mathrm{M}$ Fluo-4 (Invitrogen, USA), a fluorescent $\mathrm{Ca}^{2+}$ indicator dye, for
$30 \mathrm{~min}$ before washing once with Krebs-Ringer-HEPES

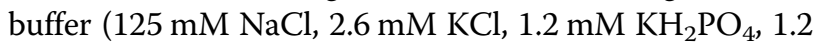
$\mathrm{mM} \mathrm{MgSO} 4 \cdot 7 \mathrm{H}_{2} \mathrm{O}, 5.5 \mathrm{mM}$ glucose, and $10 \mathrm{mM}$ HEPES, $\mathrm{pH}$ 7.4) and replenishing with Krebs-Ringer-HEPES high $\mathrm{K}^{+}$buffer (40.6 mM NaCl, $87 \mathrm{mM} \mathrm{KCl}, 1.2 \mathrm{mM} \mathrm{KH}_{2} \mathrm{PO}_{4}$, $1.2 \mathrm{mM} \quad \mathrm{MgSO}_{4} \cdot 7 \mathrm{H}_{2} \mathrm{O}, 5.5 \mathrm{mM}$ glucose, and $10 \mathrm{mM}$ HEPES, pH 7.4). Under these conditions, EGTA (final [1.25 mM]), TG (final $[1 \mu \mathrm{M}]$ ), and $\mathrm{Ca}^{2+}$ (final $[2 \mathrm{mM}]$ ) were added to the media, and the fluctuation of intracellular $\mathrm{Ca}^{2+}$ mobilization was imaged using confocal microscopy (Carl Zeiss LSM710). $\mathrm{Ca}^{2+}$ mobilization in the PAK1 stable K/D cells was also analyzed as described above.

\section{Results}

\section{PAK1 interacts with and phosphorylates STIM1}

To investigate a potential interaction with STIM1, HEK293 cells were transfected with PAK1 cDNAs alone or in combination with the STIM1 expression plasmid. The expression of myc-tagged PAK1 proteins, which included the WT, DA, and DN forms, was evaluated using an anti-myc antibody (Fig. 1), and STIM1 expression was evaluated using an anti-flag antibody. Among the three PAK1 protein forms, DN PAK1 appeared to exhibit the highest expression level regardless of STIM1 coexpression (Fig. 1a, middle row). Conversely, STIM1 expression levels increased in the presence of PAK1 (Fig. 1a, bottom row). A pull-down assay with an anti-flag (STIM1) antibody revealed that WT, DA, and DN PAK1 proteins immunoprecipitated with STIM1, indicating that STIM1 can interact with PAK1 proteins independent of its kinase activity (Fig. 1a, top row). Then, we tested the interaction between endogenous STIM1 and PAK1 using STIM1 and PAK1 antibodies, and found that both endogenous proteins interacted with each other under basal conditions. Interestingly, their interaction appeared to increase upon treatment with PDBu (Fig. 1b). To rule out the possibility of an indirect or non-specific protein-protein interaction in a human cancer cell background, such as with HeLa cells, the cytosolic region 


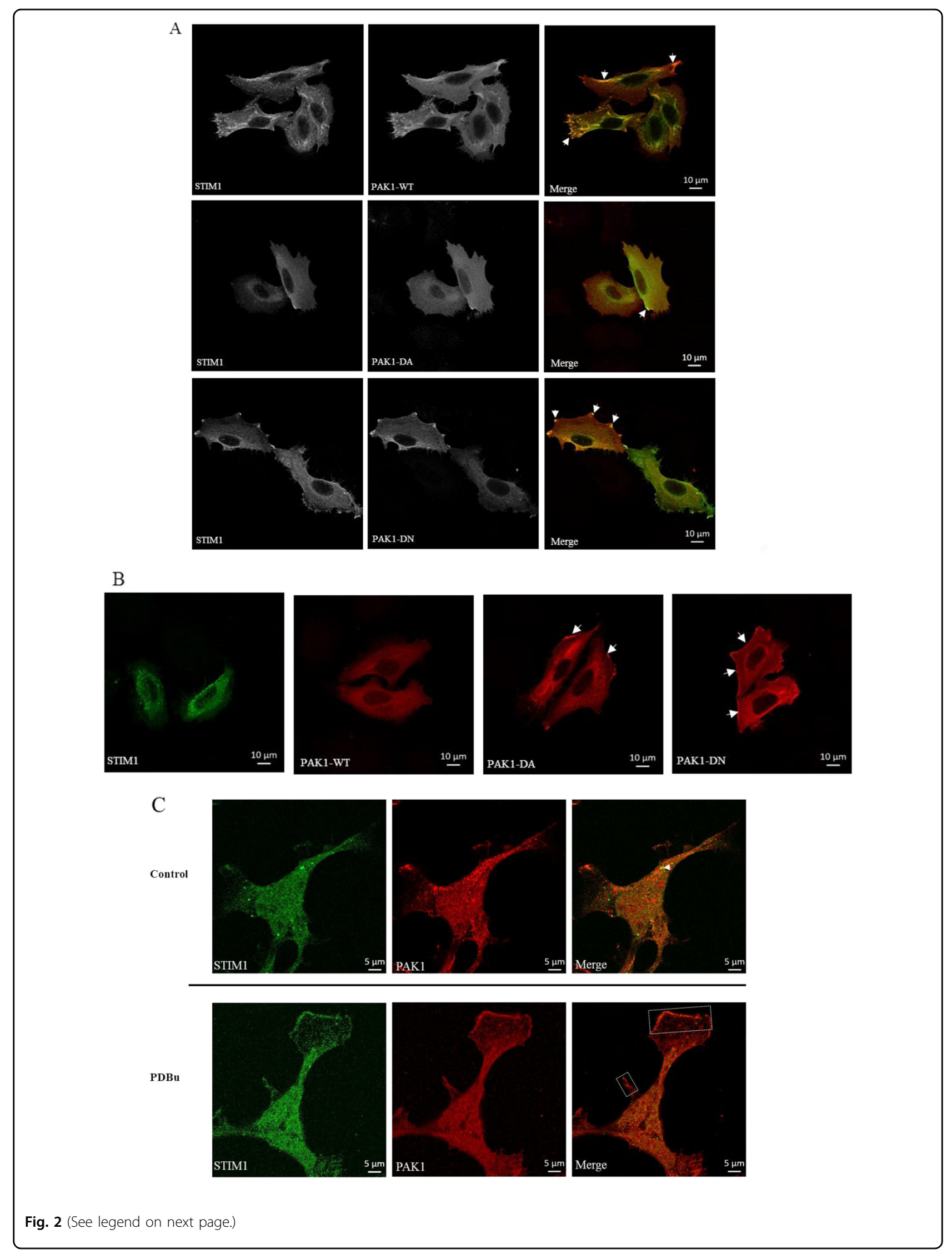


(see figure on previous page)

Fig. 2 PAK1 translocates to the cell periphery and colocalizes with STIM1. PAK1 (myc-tagged-WT, DA and DN forms) and/or STIM1 (flag taggedWT form) were expressed in HeLa cells overnight before the transfected cells were processed for confocal imaging with each primary monoclonal antibody and then with the appropriate secondary antibodies (Alexa Fluor 594- or Alexa Fluor 488-conjugated antibody, respectively), as described in the Materials and methods. The images were processed with a ZEN 2009 (Carl Zeiss). The area of colocalization (a) or lamellipodia (b) is marked with triangles. Scale bars: 5 m. Endogenous PAK1 and STIM1 were detected with antibodies against PAK1 (Abcam, rabbit monoclonal) and STIM1 (Santa Cruz, mouse monoclonal) in HEK293 cells. The areas of nascent adhesions are highlighted with white dot boxes (c). Scale bars represent 10 and $5 \mu$ m, respectively

of STIM1 was expressed as a GST fusion protein in E. coli and incubated with purified PAK1 protein overnight at $4^{\circ}$ $\mathrm{C}$ after purification, followed by washing and immunoblotting with anti-PAK1 antibody. The results of this assay revealed that STIM1 interacted with purified PAK1 as a GST fusion protein, whereas the GST protein did not pull down the PAK1 protein (Fig. 1c, d).

To further define the cytosolic region in STIM1 necessary for the interaction with PAK1, the C-terminal region of STIM1 was divided into three regions $(\mathrm{C} 1$ : spanning amino acids 235-350, C2: 341-490, and C3: 491-685) (Fig. 1e) and expressed as GST-STIM1 fusion proteins in $E$. coli (Fig. 1f). An in vitro kinase assay was performed to identify which of the three STIM1 cytosolic regions was phosphorylated by PAK1 kinase; this revealed that the $\mathrm{C} 1$ region of STIM1 may be the primary phosphorylation site and potential binding site of the PAK1 kinase (Fig. 1g). However, we were not able to further analyze the specific phosphorylation site in the $\mathrm{C} 1$ and $\mathrm{C} 2$ regions by LC-MS/MS due to a low signal-to-background noise ratio (data not shown).

\section{PAK1 colocalizes with STIM1}

PAK1 and STIM1 have been reported to exhibit different cellular localization patterns near the cell membrane depending on their activation status in the cytosol or ER, respectively. Based on our findings, we subsequently examined the localization of PAK1 and STIM1 using confocal microscopy. When overexpressed individually in HeLa cells, WT PAK1, and STIM1 each appeared primarily in the cytosol or ER, but not in the cell membrane compartment (Fig. 2b). However, the coexpression of both proteins resulted in a dramatic change in their localization patterns from the cytosol to the cell periphery or protrusions (Fig. 2a). The DA form of PAK1 primarily localized in the cytosol with some expression in the cell periphery, whereas co-expression with STIM1 resulted in the colocalization of both proteins in the cytosol; the overall cell morphology appeared similar to cells expressing only the PAK1 mutant. In contrast, the DN form of PAK1 induced a cell shape with lamellipodia, whereas the co-expression of PAK1 and STIM1 resulted in colocalization of both proteins at cell adhesions. Under basal conditions, endogenous PAK1 and STIM1 colocalized in the cytosol, but treatment with $\mathrm{PDBu}$ caused a shift in the localization patterns of both proteins to include the cell periphery and protrusions (Fig. 2c).

\section{The level of intracellular $\mathrm{Ca}^{2+}$ mobilization correlates with PAK1 activity}

Next, we attempted to determine whether PAK1 modulates SOCE activity. Accordingly, HeLa cells were transfected with kinase-active and -inactive forms of PAK1 cDNAs in a RFP plasmid and loaded with Fluo-4 for $\mathrm{Ca}^{2+}$ imaging using confocal microscopy. The depletion of $\mathrm{ER} \mathrm{Ca}^{2+}$ was induced with TG, and SOCE activation was analyzed after the addition of $2 \mathrm{mM} \mathrm{Ca}^{2+}$. As shown in Figure 3a, the kinase-active form of PAK1 exhibited increased $\mathrm{Ca}^{2+}$ mobilization after both TG stimulation and SOCE activation compared to the RFP vector control, whereas the kinase inactive form of PAK1 appeared to inhibit $\mathrm{Ca}^{2+}$ mobilization. To confirm this finding, we used chemical inhibitors of PAK1, including 1, 4-naphthohydroquinone (1, 4-NHQ) and PF3758309, in the $\mathrm{Ca}^{2+}$ assay. As shown in Figure 3b, c, treatment with both PAK1 inhibitors prevented TG-induced $\mathrm{Ca}^{2+}$ mobilization in a dose-dependent manner. Additionally, the localization of WT STIM1 and WT PAK1 changed following treatment with TG from cell protrusions or puncta in the cytosol to stress fiber-like structures (Fig. 3d).

We also established a PAK1 K/D HeLa cell line expressing PAK1 siRNA and analyzed whether PAK1 expression levels were correlated with SOCE-mediated $\mathrm{Ca}^{2+}$ mobilization levels. As shown in Figure 3e, the PAK1 K/D cell line exhibited decreased PAK1 levels and reduced SOCE activity compared to the control (scrambled) HeLa cell line (Fig. 3e), clearly suggesting the significance of optimal PAK1 levels for the SOCE process.

\section{PAK1 and STIM1 localize at vinculin-positive cell protrusions and nascent adhesions}

To characterize the nature of PAK1 and STIM1 localization at cell protrusions and the cell periphery, HeLa cells were transfected with PAK1 cDNAs (c-Myc-tagged) and/or eGFP-STIM1. The transfected cells were treated with $\mathrm{PDBu}$ to elicit a change in cell adhesion before processing the cells using anti-c-myc (PAK1) and anti- 
A

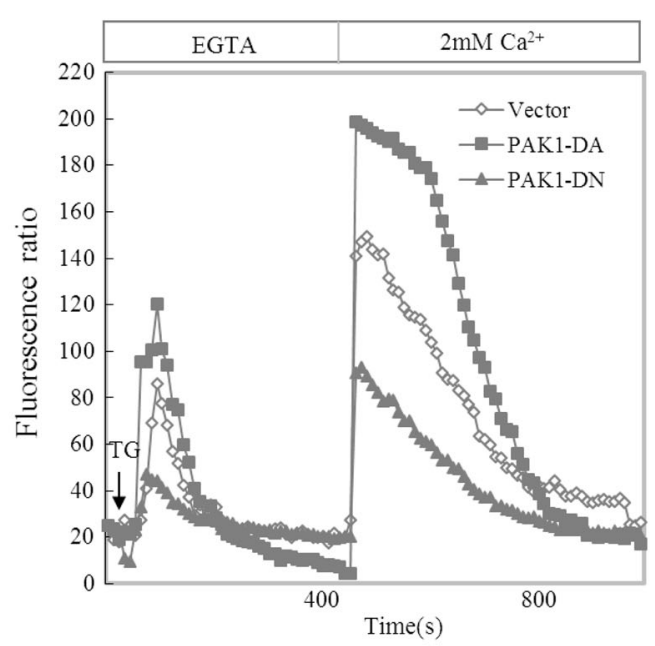

C

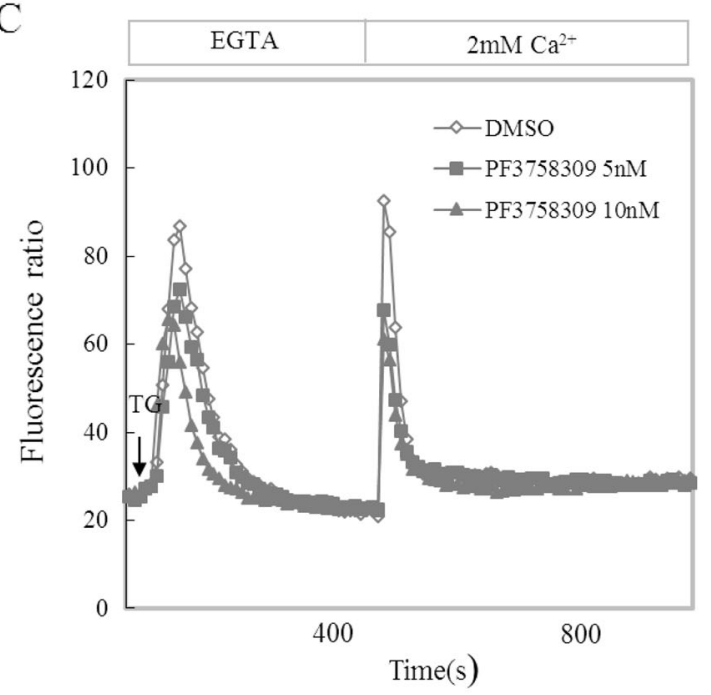

B

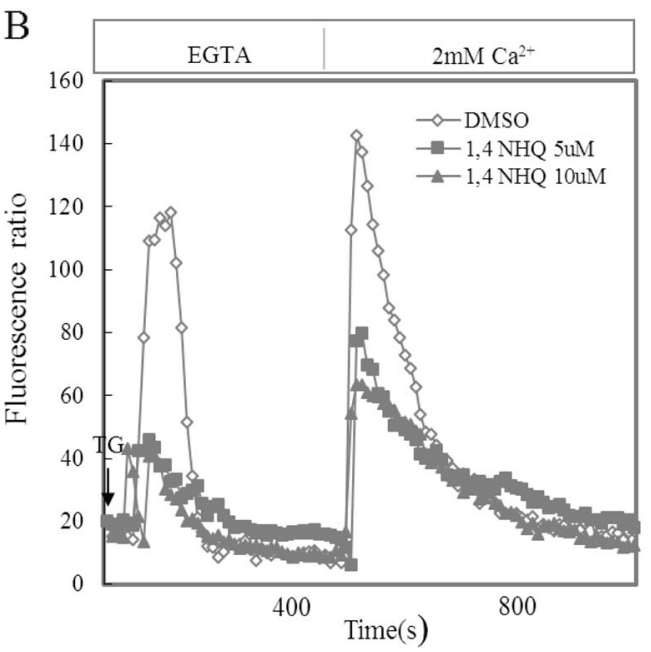

D
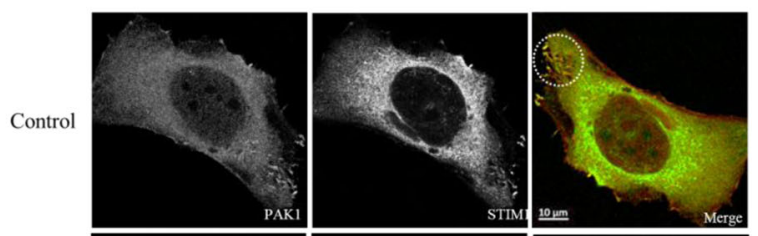

TG

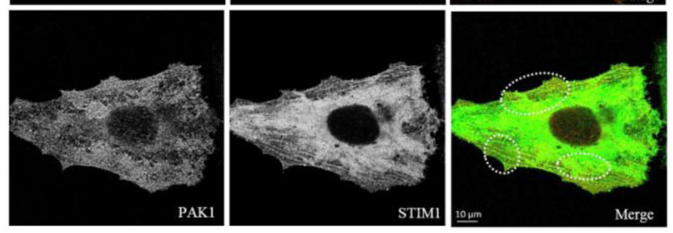

E

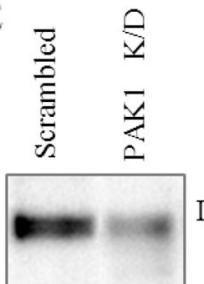

IB: PAK1

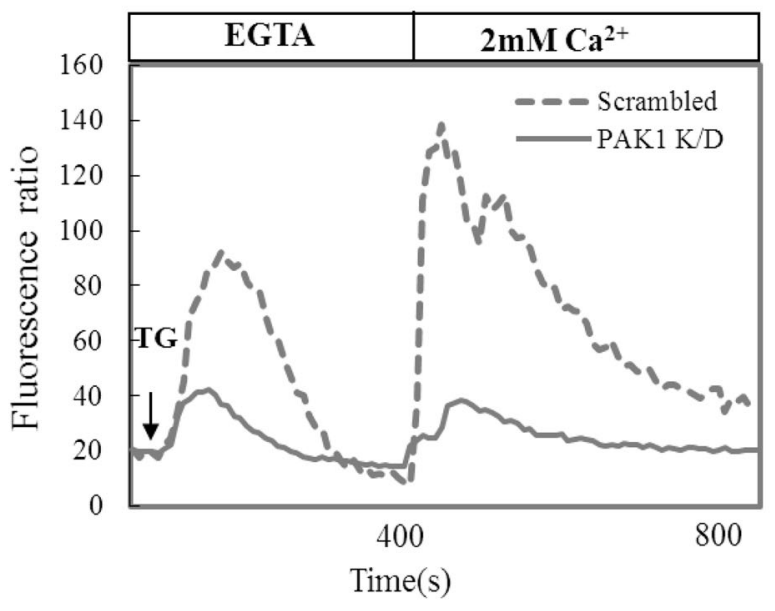

Fig. 3 (See legend on next page.) 
(see figure on previous page)

Fig. 3 The level of intracellular $\mathrm{Ca}^{2+}$ mobilization correlates with PAK1 activity. Active or inactive forms of PAK1 CDNA in the RFP plasmid were transfected into HeLa cells; after an overnight incubation, these cells were loaded with $1 \mu \mathrm{M}$ Fluo-4 for calcium imaging using confocal microscopy. ER calcium depletion was induced with $1 \mu \mathrm{M} \mathrm{TG}$, and thereafter, SOCE activation was analyzed after the addition of $2 \mathrm{mM} \mathrm{Ca}^{2+}$ (a). In addition, HeLa cells were pretreated with chemical inhibitors of PAK1, such as 1,4-NHQ (5 or $10 \mu \mathrm{M}, \mathbf{b})$ or PF3758309 (5 or $10 \mathrm{nM}$, c), before the calcium assay. Additionally, the localization of overexpressed STIM1 and PAK1 was determined after TG treatment and the addition of exogenous calcium by confocal microscopy - the area of colocalization is circled (d). PAK1 knockdown cells were established using HeLa cells via transfection of the shDNA plasmid against PAK1, and after western blot analysis of the reduced levels of PAK1 expression, control scramble and PAK1 knockdown cell lines were assayed for calcium mobilization (e)

vinculin antibodies for confocal imaging. In control cells, vinculin localized to stable focal adhesions, whereas STIM1 and PAK1 did not appear to colocalize with vinculin. However, after modulating the focal adhesions with $\mathrm{PDBu}$ treatment, vinculin colocalized with PAK1 and STIM1 at nascent adhesions on the cell periphery and cell protrusions (Fig. 4a). Conversely, when PAK1 was overexpressed alone, it did not colocalize with the vinculin marker protein at the focal adhesions, whereas PDBu treatment induced numerous nascent adhesions to which PAK1 and vinculin colocalized (Fig. 4b control). Under control basal conditions, eGFP-STIM1 appeared to localize primarily in the cytosol and rarely colocalized with vinculin in the cell periphery. In PDBu-treated cells, some degree of eGFP-STIM1 colocalizations with vinculin was found in the cell periphery.

\section{Discussion}

PAK1 is known to control cellular focal adhesion strength by regulating the distribution of myosin IIA and actin dynamics to optimize cell migration ${ }^{33}$ and cell adhesion ${ }^{40}$. Furthermore, these biological roles are known to require precise spatial and kinetic control of PAK1 localization and activity ${ }^{28,41}$.

Our study demonstrated that PAK1 interacts with STIM1 in vitro under endogenous and overexpression conditions and that PAK1 activity does not appear to be important in the interaction. The results of the in vitro kinase assay suggested that the $\mathrm{C} 1$ and $\mathrm{C} 2$ domains of STIM1 might be overlapping or separate sites for the interaction with and/or the phosphorylation by PAK1. However, the C3 C-terminal end domain, which is 195 amino acids long and contains the site of ERK1/2 phosphorylation for EB1 dissociation and SOCE activation ${ }^{42}$, was not phosphorylated by PAK1. This could suggest that the PAK1 and MAPK signaling pathways regulate STIM1 differently by targeting different domains of STIM $1^{43}$. Hence, it will be interesting to determine whether PAK1 can phosphorylate STIM1 irrespective of the $\mathrm{Ca}^{2+}$ influx due to the activation of SOCE, as seen with ERK $1^{43}$.

PAK1 reportedly localizes primarily in the cytosol in an inactive form; the activation of PAK1, via interaction with the activators Cdc42-GTP and Rac1-GTP, promotes its localization at cellular protrusions ${ }^{41}$. As noted previously,
STIM1 translocates from the cytosol near the PM by binding to EB1, thus connecting the tubular ER directly to growing microtubules toward the ER-PM junction ${ }^{9,}{ }^{10}$. However, how these GTPases and/or their downstream effector PAK1 regulate $\mathrm{Ca}^{2+}$ mobilization has been unclear.

Our confocal data revealed that STIM1 and PAK1 primarily localized in the cytoplasm, whereas PDBu treatment distinctly induced their colocalization at nascent adhesions, suggesting that both proteins were activated. How these two proteins exhibit such a colocalization pattern remains unclear, as well as whether a reciprocal control mechanism exists between the two proteins or unilateral regulation by one protein over the other. Precise PAK1 kinase activity might be important, as only WT PAK1, but not the DA or DN PAK1 mutants, induced nascent adhesions similar in size and number to the nascent adhesions of PDBu-treated cells.

We were interested in characterizing the nature of the cellular protrusions to which PAK1 and STIM1 colocalized. Under basal conditions, neither protein colocalized with vinculin at the cell periphery, and both were found in the cytosol. However, when the cells were treated with $\mathrm{PDBu}$ to modulate stable focal adhesions for nascent adhesions, both proteins appeared to colocalize at vinculin-positive nascent adhesions at the cell periphery but not in the stable focal adhesions (Fig. 4). This result indicated that PAK1 and STIM1 might transiently interact and colocalize in nascent adhesions before the formation of stable focal adhesions.

Taken together, our data demonstrate that PAK1 interacts with STIM1 and phosphorylates specific STIM1 cytosolic domains. Based on the $\mathrm{Ca}^{2+}$ assay data, PAK1 appears to play a role in modulating the STIM1-mediated SOCE process. The PDBu confocal data suggest that PAK1 and STIM1 transiently migrate from the cytosol to the cell periphery in nascent adhesions. However, further studies are required to determine the exact role PAK1 and STIM1 play in the formation of nascent adhesions with respect to Rho family GTPases and whether there is an optimal $\mathrm{Ca}^{2+}$ concentration window for the formation of these transient adhesion structures. Additional studies are also needed to elucidate the role of PAK1 in cancer cell migration and metastasis. 


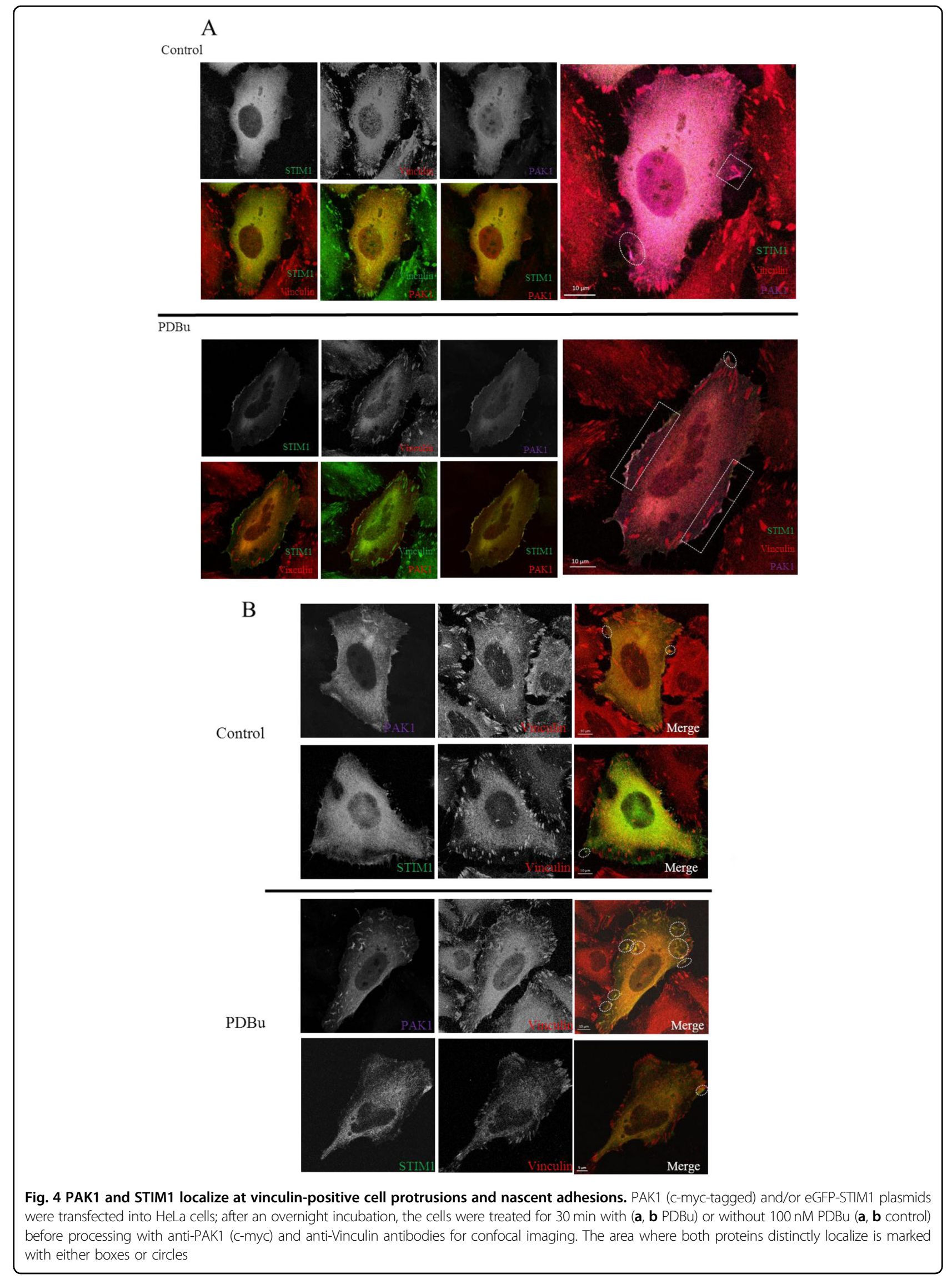




\section{Acknowledgements}

This work was supported by the Next Generation BioGreen21 project (Project No. PJ01334802, PJ01104401 and PJ01110901) of the Rural Development Administration, Republic of Korea. This work was also supported by a National Research Foundation of Korea (NRF) grant funded by the South Korean government (MSIP) (NO. MRC, 2017R1A5A2015541).

\section{Author details}

'Division of Biochemistry, Chungbuk National University, City of Cheongju 361763 , Korea. ${ }^{2}$ Department of Pediatrics, College of Medicine, Chungbuk National University, City of Cheongju 361-763, Korea. ${ }^{3}$ College of Pharmacy and Medical Research Center, Chungbuk National University, City of Cheongju 361-763, Korea. ${ }^{4}$ Department of Animal Biotechnology, Chonbuk National University, Jeonju-si, Jeollabuk-do 54896, Korea

\section{Conflict of interest}

The authors declare that they have no conflict of interest.

\section{Publisher's note}

Springer Nature remains neutral with regard to jurisdictional claims in published maps and institutional affiliations.

Received: 14 August 2017 Revised: 13 February 2018 Accepted: 13 March 2018.

Published online: 21 May 2018

\section{References}

1. Marchant, J. S. Cellular signalling: STIMulating calcium entry. Curr. Biol. 15, R493-R495 (2005).

2. Cahalan, M. D. et al. Molecular basis of the CRAC channel. Cell Calcium 42 133-144 (2007).

3. Collins, S. R. \& Meyer, T. Evolutionary origins of STIM1 and STIM2 within ancient $\mathrm{Ca}^{2+}$ signaling systems. Trends Cell Biol. 21, 202-211 (2011).

4. Prakriya, M. \& Lewis, R. S. Store-operated calcium channels. Physiol. Rev. 95 1383-1436 (2015)

5. Moccia, F. et al. Stim and Orai proteins in neuronal $\mathrm{Ca}\left({ }^{2+}\right)$ signaling and excitability. Front. Cell. Neurosci. 9, 153 (2015).

6. Zhang, S. L. et al. STIM1 is a $\mathrm{Ca}^{2+}$ sensor that activates CRAC channels and migrates from the $\mathrm{Ca}^{2+}$ store to the plasma membrane. Nature 437, 902-905 (2005).

7. Liou, J. et al. STIM is a $\mathrm{Ca}^{2+}$ sensor essential for $\mathrm{Ca}^{2+}$-store-depletion-triggered $\mathrm{Ca}^{2+}$ influx. Curr. Biol. 15, 1235-1241 (2005).

8. Lee, J. E. et al. Ubiquilin 1 interacts with Orai1 to regulate calcium mobilization. Mol. Cells 35, 41-46 (2013).

9. Grigoriev, I. et al. STIM1 is a MT-plus-end-tracking protein involved in remodeling of the ER. Curr. Biol. 18, 177-182 (2008).

10. Jermy, A. STIM1 tracks growing microtubule ends. Nat. Cell. Biol. 10, 384 (2008),

11. Honnappa, S. et al. An EB1-binding motif acts as a microtubule tip localization signal. Cell 138, 366-376 (2009).

12. Okeke, E., Dingsdale, H., Parker, T., Voronina, S. \& Tepikin, A. V. Endoplasmic reticulum-plasma membrane junctions: structure, function and dynamics. J. Physiol. 594, 2837-2847 (2016).

13. Patterson, R. L., van Rossum, D. B. \& Gill, D. L. Store-operated Ca21 entry evidence for a secretion-like coupling model. Cell 98, 487-499 (1999).

14. Galan, C., Dionisio, N., Smani, T., Salido, G. M. \& Rosado, J. A. The cytoskeleton plays a modulatory role in the association between STIM1 and the $\mathrm{Ca}^{2+}$ channel subunits Orai1 and TRPC1. Biochem. Pharmacol. 82, 400-410 (2011).

15. $\mathrm{Xu}, \mathrm{Y}$. et al. STIM1 accelerates cell senescence in a remodeled microenvironment but enhances the epithelial-to-mesenchymal transition in prostate cancer. Sci. Rep. 5, 11754 (2015).

16. Wang, J. Y. et al. STIM1 overexpression promotes colorectal cancer progression, cell motility and COX-2 expression. Oncogene 34, 4358-4367 (2015).

17. Zhu, M. et al. Store-operated $\mathrm{Ca}^{\left({ }^{2+}\right)}$ entry regulates glioma cell migration and invasion via modulation of Pyk2 phosphorylation. J. Exp. Clin. Cancer Res. 33, 98 (2014).

18. Kim, J. H. et al. Orai1 and STIM1 are critical for cell migration and proliferation of clear cell renal cell carcinoma. Biochem. Biophys. Res. Commun. 448, 76-82 (2014).
19. Casas-Rua, V. et al. STIM1 phosphorylation triggered by epidermal growth factor mediates cell migration. Biochim. Biophys. Acta 1853, 233-243 (2015).

20. Che, $\mathrm{H}$. et al. Roles of store-operated $\mathrm{Ca}^{2+}$ channels in regulating cell cycling and migration of human cardiac c-kit + progenitor cells. Am. J. Physiol. Heart Circ. Physiol. 309, 1772-1781 (2015).

21. Tapon, N. \& Hall, A. Rho, Rac and Cdc42 GTPases regulate the organization of the actin cytoskeleton. Curr. Opin. Cell Biol. 9, 86-92 (1997).

22. Daniels, R. H. \& Bokoch, G. M. p21-Activated protein kinase- a crucial component of morphological signaling. Trends Biochem. Sci. 24, 350-355 (1999).

23. Hall, A. Rho GTPases and the control of cell behaviour. Biochem. Soc. Trans. 33 891-895 (2005).

24. Bustelo, X. R., Sauzeau, V. \& Berenjeno, I. M. GTP-binding proteins of the Rho Rac family regulation, effectors and functions in vivo. Bioessays $\mathbf{2 9}, 356-370$ (2007).

25. Sells, M. A. et al. Human p21-activated kinase (Pak1) regulates actin organization in mammalian cells. Curr. Biol. 7, 202-210 (1997).

26. Bishop, A. L. \& Hall, A. Rho GTPases and their effector proteins. Biochem. J. 1, 241-255 (2000)

27. Zhao, Z. S. \& Manser, E. PAK and other Rho-associated kinases-effectors with surprisingly diverse mechanisms of regulation. Biochem. J. 386, 201-214 (2005).

28. Stofega, M. R., Sanders, L. C., Gardiner, E. M. \& Bokoch, G. M. Constitutive p21activated kinase (PAK) activation in breast cancer cells as a result of mislocalization of PAK to focal adhesions. Mol. Biol. Cell 15, 2965-2977 (2004).

29. Itakura, A. et al. p21-Activated kinase (PAK) regulates cytoskeletal reorganization and directional migration in human neutrophils. PLOS ONE 8, e73063 (2013).

30. Abo, A. et al. PAK4, a novel effector for $\mathrm{Cdc} 42 \mathrm{Hs}$, is implicated in the reorganization of the actin cytoskeleton and in the formation of filopodia. EMBO J. 17, 6527-6540 (1998).

31. Brown, M. C., West, K. A. \& Turner, C. E. Paxillin-dependent paxillin kinase linker and p21-activated kinase localization to focal adhesions involves a multistep activation pathway. Mol. Biol. Cell 13, 1550-1565 (2002).

32. Loo, T. H., Ng, Y. W., Lim, L. \& Manser, E. GIT1 activates p21-activated kinase through a mechanism independent of p21 binding. Mol. Cell. Biol. 24, 3849-3859 (2004).

33. Delorme-Walker, V. D. et al. Pak1 regulates focal adhesion strength, myosin IIA distribution, and actin dynamics to optimize cell migration. J. Cell Biol. 193, 1289-1303 (2011).

34. Aslan, J. E. et al. The PAK system links Rho GTPase signaling to thrombinmediated platelet activation. Am. J. Physiol. Cell Physiol. 305, C519-C528 (2013).

35. Allen, J. D. et al. p21- activated kinase regulates mast cell degranulation via effects on calcium mobilization and cytoskeletal dynamics. Blood 113, 2695-2705 (2009)

36. Kosoff, R., Chow, H. Y., Radu, M. \& Chernoff, J. Pak2 kinase restrains mast cell FceRl receptor signaling through modulation of Rho protein guanine nucleotide exchange factor (GEF) activity. J. Biol. Chem. 288, 974-983 (2013).

37. Nuche-Berenguer, B. \& Jensen, R. T. Gastrointestinal hormones/neurotransmitters and growth factors can activate P21 activated kinase 2 in pancreatic acinar cells by novel mechanisms. Biochim. Biophys. Acta 1853, 2371-2382 (2015).

38. Wang, Y. et al. Pak1 is required to maintain ventricular $\left.\mathrm{Ca}^{2+}{ }^{2+}\right)$ homeostasis and electrophysiological stability through SERCA2a regulation in mice. Circ. Arrhythm. Electrophysiol. 7, 938-948 (2014).

39. Kim, D. J. et al. Small molecules that allosterically inhibit p21-activated kinase activity by binding to the regulatory p21-binding domain. Exp. Mol. Med. 48, e229 (2016).

40. Eby, J. J. et al. Actin cytoskeleton organization regulated by the PAK family of protein kinases. Curr. Biol. 8, 967-970 (1998).

41. Parrini, M. C., Matsuda, M. \& de Gunzburg, J. Spatiotemporal regulation of the Pak1 kinase. Biochem. Soc. Trans. 33, 646-648 (2005).

42. Soboloff, J., Madesh, M. \& Gill, D. L. Sensing cellular stress through STIM proteins. Nat. Chem. Biol. 7, 488-492 (2011).

43. Tomas-Martin, P., Lopez-Guerrero, A. M., Casas-Rua, V., Pozo-Guisado, E. \& Martin-Romero, F. J. Phospho-STIM1 is a downstream effector that mediates the signaling triggered by IGF-1 in HEK293 cells. Cell. Signal. 27, 545-554 (2015). 